\title{
Investigation of the incoming wind vector for improved wind turbine yaw-adjustment under different atmospheric and wind farm conditions
}

\author{
G. Cortina ${ }^{a}$, V. Sharma ${ }^{b}$, M. Calaf ${ }^{a}$ \\ ${ }^{a}$ Department of Mechanical Engineering, University of Utah, Utah, USA \\ ${ }^{b}$ School of Architecture, Civil and Environmental Engineering, EPFL, Lausanne, Switzerland
}

\begin{abstract}
Regardless of the evolution of wind energy harvesting, the way in which turbines obtain in-situ meteorological information remains the same - i.e. using traditional wind vanes and cup anemometers installed at the turbine's nacelle, right behind the blades. As a result, misalignment with the mean wind vector is common and energy losses up to $4.6 \%$ can be experienced as well as increases in loading and structural fatigue. A solution for the nearblade monitoring is to install wind LIDAR devices on the turbines' nacelle. This technique is currently under development as an alternative to traditional in-situ wind anemometry because it can measure the wind vector at substantial distances upwind. But at what upwind distance should they interrogate the atmosphere? and, what is the optimal average time in which to learn about the incoming flow conditions? This work simulates wind fields approaching isolated wind turbines and wind turbine arrays within large wind farms using Large Eddy Simulations. The goal is to investigate the existence of an optimal upstream scanning distance and average time for wind turbines to measure the incoming wind conditions under different ambient atmospheric conditions. Results reveal no significant differences when measuring the incoming wind vector at different upstream distances, regardless of the atmospheric stratification. Within this framework a 30 minutes readjustment period is observed to perform the best.
\end{abstract}

Keywords: Large Eddy Simulation, LIDAR, Wind Energy, Wind Farm, Wind Turbines, Yaw-misalignment

\section{Introduction}

Over the past ten years wind energy harvesting has experienced a rapid growth, with 3 an overall installed global power average annual increase of the total installed wind power 4 capacity of $25 \%$ per year in the last ten years (2002-2012) [1]. Throughout this time wind

Email address: marc.calaf@utah.com (M. Calaf)

URL: http://wet.mech.utah.edu/ (M. Calaf)

Preprint submitted to Renewable Energy

May 31, 2016 
turbine designs have evolved, with increases in rotor diameter and overall efficiency designs. By 2004-08 most wind turbines had a $70-80$ meter rotor diameter, and currently, rotor diameter designs are approaching 100 meters with an average hub height of 80 meters [2]. Engineering improvements have accompanied the development of large rotors. However, the way in which wind turbines obtain in-situ meteorological information remains the same - traditional wind vanes and cup anemometers are installed at the turbines nacelle, right behind the blades. The orientation and other operating parameters of the turbine are governed by these two wind measurements through the use of a control loop [3].

These wind measurements have important drawbacks, especially with increasing rotor diameters. Since the velocity is measured at a single point immediately behind the blades, turbine misalignment with the mean wind direction is common [4]. In principle, this problem should be overcome using the so-called nacelle transfer functions (NTFs), which describe the relation between the nacelle wind vector measurements and the unperturbed wind vector upstream of the wind farm. The NTFs are used to properly correct nacelle measurements [5]; however, they have a strong variability from site to site and they don't account for changes in atmospheric-stratification. For example, Vanderwende and Lundquist [6] experimentally showed distinct wind turbine performances, with respect to a given wind turbine power coefficient curve, under different atmospheric regimes. They demonstrated under-performance during stably-stratified periods and over-performance during unstably-stratified periods and moderate wind speeds $\left(8-12 \mathrm{~ms}^{-1}\right)$. An additional drawback from the near-blade monitoring is the limited time it provides the wind turbine controllers to adjust the turbine operating parameters' to account for changes in the approaching wind flow [7]. This latency in adjusting to ambient wind direction and speed subjects the blades and turbine structure to unanticipated wind gusts or extreme incoming wind conditions. These velocity aberrations induce increased loading, structural fatigue, power-train failures and associated increases in maintenance costs. A solution is to install forward directed wind Laser Imaging Detection and Ranging (LIDAR) on the turbines' nacelles. Future studies might find a way to measure ahead of time these velocity aberrations and wind gusts with the use of wind LIDARs, which together with advanced control systems will advice the wind turbine controls system to correct their settings. Also experimental LES studies should be performed in order to study the turbulent flow upstream of the wind turbines. Although LIDAR technology has been around since the early 1970s [8, 9, 10], it hasn't been until the recent advancements in optical fiber technology that such LIDARs have become affordable. Because wind LIDARs measure the frequency shift of the backscattered light initially sent by a laser beam, making it possible to measure the along-beam component of the wind velocity at various distances upwind of the rotor. By using a cone-scanning or volumetric scanning technique, the full three-dimensional wind components can be used to measure the approaching unperturbed winds $[7,11,12,13,14,15,16]$.

Further, recent studies have shown the potential of optimizing the overall power output of a wind farm by forcing a fixed yaw-misalignement on the upwind wind turbine arrays such that their corresponding wakes are laterally deviated and reducing their corresponding interaction with the downstream turbines [17, 18, 19]. Gebraad et al. [18] developed a control-oriented dynamic model that determines the best orientation of a given wind turbine 
to avoid turbine-wake interaction. Also the National Renewable Energy Laboratory (NREL) experimentally implemented at the Fishermens Atlantic City Windfarm a control strategy to reduce the wind-turbine wake interaction, obtaining up to a $10 \%$ increase in harvested power for the overall wind farm [19]. Yet, for these wind farm optimization techniques to be efficient, it is critical to accurately know the correct yaw-aligmment of the turbine with respect to the incoming wind vector. The use of a wind LIDAR mounted on the turbine's nacelle has been shown to provide good yaw alignment and better power curves than traditional wind vanes, therefore enhancing the overall power output [20,21, 22]. However, wind LIDAR systems are optimized to measure over a fixed upwind range and average over a certain period of time. The question remains as to what upwind distance they should interrogate the atmosphere and for what time period. In the present work, different upstream scanning distances and averaging times are evaluated within different atmospheric stratifications using Large Eddy Simulations (LES) driven with the experimental data of the well known Cooperative Atmospheric Surface Exchange Study (CASES-99) [23, 24, 25]. In section 2 , the LES code is presented in detail together with the sub-grid model, the boundary conditions and the wind turbine model used. Section 3 introduces the study cases considered, and section 4 introduces the numerical results, first with respect to different upstream scanning distances and second with respect to distinct yawing averaging times. Finally, the conclusions are presented in section 5 .

\section{Large-Eddy Simulation Framework}

The present work uses the Large Eddy Simulation code introduced in Sharma et al.[26]. The numerical code integrates the non-dimensional, incompressible, and filtered NavierStokes (NS) equations together with the continuity equation. The NS equation is implemented using its rotational form to assure conservation of energy and mass of the inertial terms [27]. The effect of temperature is introduced by means of a buoyancy term in the NS equation, which is the result of the Boussinesq approximation, and which is obtained from solving a coupled advection-diffusion equation for the potential temperature $(\theta)$. Therefore, the full set of equations solved is,

$$
\begin{gathered}
\frac{\partial \tilde{u}_{i}}{\partial x_{i}}=0 \\
\frac{\partial \tilde{u}_{i}}{\partial t}+\tilde{u}_{j}\left(\frac{\partial \tilde{u}_{i}}{\partial x_{j}}-\frac{\partial \tilde{u}_{j}}{\partial x_{i}}\right)=-\frac{1}{\rho} \frac{\partial p^{*}}{\partial x_{i}}-\frac{\partial \tilde{\tau}_{i j}}{\partial x_{j}}+g\left(\frac{\tilde{\theta}-\langle\tilde{\theta}\rangle}{\theta_{0}}\right) \delta_{i 3}+ \\
+f\left(\tilde{u}_{2}-v_{G}\right) \delta_{i 1}-f\left(\tilde{u}_{1}-u_{G}\right) \delta_{i 2}+f_{i}, \\
\frac{\partial \tilde{\theta}}{\partial t}+\tilde{u}_{j} \frac{\partial \tilde{\theta}}{\partial x_{j}}=-\frac{\partial \pi_{j}}{\partial x_{j}} .
\end{gathered}
$$

Here, the tilde $(\sim)$ represents the LES filtering operation at the grid-size $\Delta$, and the angle brackets $(\langle\cdot\rangle)$ represent a horizontal average, with index notation used to specify rectangular 
Cartesian coordinates $i=1,2,3=x, y, z$. The reference temperature is denoted by $\theta_{0}$. The $\tilde{\tau}_{i j}$ term represents the deviatoric part of the momentum sub-grid stress (SGS) term, which is modeled using the Lagrangian Scale Dependent model of Bou-Zeid et al. [28], and $\pi_{j}$ represents the sub-grid component of the thermal equation, which is correspondingly modeled with the adaptation of the Lagrangian Scale Dependent model for scalars introduced in Calaf et al. [29]. The modified kinematic pressure term $\left(p^{*}\right)$ includes the filtered pressure term and the trace of the SGS tensor $\left(\tilde{p} / \rho+\tilde{\tau}_{k k} / 3+\frac{1}{2} \tilde{u}_{j} \tilde{u}_{j}\right)$. Within this work, the flow is forced with a time- and height-independent geostrophic wind $\left(u_{G}, v_{G}\right)$, where $f$ represents the Coriolis parameter, and $\delta_{i j}$ is the Kronecker delta $\left(\delta_{i j}=1\right.$, if $i=j$, and 0 otherwise). The $f_{i}$ term represents the sink of momentum induced by the wind turbine. It is represented as a body force (per unit volume) and it is modeled using the traditional actuator-disk with rotation (ADR, see Wu et al. [30]) including the dynamical yaw-alignement of Sharma et al. [26]. Note that within the formulation of these equations the potential temperature is an active scalar that modifies the momentum equation by means of the buoyancy term, which accounts for the vertical motions induced by the thermal stratification. Further, as it is traditional in LES of atmospheric flows, the viscous effects are neglected and the flow is therefore characterized by a very large Reynolds number. The numerical discretization of the equations follows the one introduced by Moeng [31] and Alberston et al. [32], where a pseudospectral approach with a staggered-grid is used. Therefore, second-order finite differences are used in the vertical direction and a spectral discretization using the Fast Fourier Transform in the West (FFTW) [33] library is implemented in the horizontal directions. As a result of the Fourier discretization in the horizontal directions the numerical domain becomes periodic, eliminating the need for lateral boundary conditions and becoming infinite in practical effects.

The equations are dealiazed using the 3/2-rule [34], and time-integrated using a second order Adam-Bashfort scheme. The numerical algorithm is fully parallelized using the Message-Passing Interface (MPI) with a total of 64 processors and the pressure solver is further parallelized with the pipeline Thomas algorithm [35]. As a result of the periodic boundary conditions in the horizontal directions, there is no need for lateral boundary conditions. For the top boundary a zero-flux and zero-shear are imposed, with the vertical velocity and the gradients of the horizontal velocities equal to zero. At the surface, the non-slip condition is imposed for the vertical velocity, and because of the staggered grid, an equivalent shear stress is imposed at the first grid point for the horizontal velocities. The shear stress at the surface is parametrized using the traditional log-law including the effects of surface stratification [36, 28, 37],

$$
\tau_{i, 3}\left(x, y, z_{1}\right)=-\left[\frac{k \sqrt{\left(\hat{\tilde{u}}_{1}^{2}+\hat{\tilde{u}}_{2}^{2}\right)}}{\ln \left(z_{1} / z_{0}\right)+\psi_{m}\left(z_{1} / L\right)}\right]^{2} n_{i} .
$$

In this equation $z_{1}$ indicates the height of the first grid point where the horizontal velocity components are computed $\left(\Delta_{z} / 2\right)$ and where the shear stress is applied, $i$ indicates any of the horizontal plane-parallel directions $(i=1,2)$, and $n_{i}$ is a unitary directional vector, 
$n_{i}=\hat{\tilde{u}}_{i} / \sqrt{\hat{\tilde{u}}_{1}^{2}+\hat{\tilde{u}}_{2}^{2}}$. While this expression was originally developed for ensemble averages and later interpreted as spatial averages over homogeneous surfaces, here a double filtering at $2 \Delta$ grid spacing, equivalent to a local averaging, is used instead (see $[28,37]$ for further details in this filtering). In this study the surface roughness is maintained homogeneous over the entire domain with a value of $z_{0}=3 \cdot 10^{-5} z_{i}$ (where $z_{i}$ is the initial inversion height, which will be used as a normalization length-scale, see later). To numerically integrate the NS equations, the vertical derivatives of the horizontal velocities are also parametrized at the first grid point $\left(z_{1}\right)$ using Monin-Obukhov similarity theory [38],

$$
\partial_{3} \tilde{u}_{i}\left(x, y, z_{1}\right)=\left(\frac{\sqrt{\tau}}{\kappa z}\right) n_{i}
$$

with $\tau=\sqrt{\tau_{1,3}^{2}+\tau_{2,3}^{2}}$. In addition, to integrate the advection-diffussion equation for the potential temperature a sensible heat flux is imposed at the first grid point, similar to the momentum, also using Monin-Obukhov similarity theory,

$$
H_{s}\left(x, y, z_{1}\right)=\frac{\kappa^{2}\left[\theta_{s}-\tilde{\theta}\left(x, y, z_{1}\right)\right]\left(\sqrt{\hat{\tilde{u}}_{1}^{2}+\hat{\tilde{u}}_{2}^{2}}\right)}{\left[\ln \left(\frac{z_{1}}{z_{0}}\right)+\psi_{m}(z / L)\right]\left[\ln \left(\frac{z_{1}}{z_{0, h}}\right)+\psi_{h}(z / L)\right]} .
$$

The surface temperature $\left(\theta_{s}\right)$ is imposed as bottom boundary condition (see Figure 1a) and $z_{0, h}$ represents the scalar surface roughness, which has been taken to be one tenth of the momentum surface roughness $\left(z_{0, h}=z_{0} / 10\right)$ [39]. The stability correction functions $(\psi(z / L))$ implemented are those from Brutsaert [40]. Different formulations exist depending on stability and the appropriate choice of functions is made based upon the Obukhov length (L) depicted in Figure 1b and defined as

$$
L=\frac{-u_{\star}^{3} \bar{\theta}_{s}}{\kappa g\left(\overline{w^{\prime} \theta^{\prime}}\right)_{s}},
$$

where $u_{\star}$ is the friction velocity, $\bar{\theta}$ is the mean potential temperature, $\kappa$ is the von Káráman constant $(\kappa=0.4), g$ represents the gravity term and $\overline{w^{\prime} \theta^{\prime}}$ is the surface sensible heat flux. In this work it is well understood that the stability correction functions were initially developed from experimental studies on statistically homogeneous surfaces and that the wind turbines might have an effect on the precise parametrization of the stability correction functions. However, lack of new experimental data on this precise matter doesn't allow for a better numerical approach at the present time.

\section{Study case description}

To develop this study two different sets of simulations, both of which included the presence of wind turbines, were considered. One case consisted of a traditional very large wind farm (VLWF), and the second one consisted of a largely spaced wind farm (LSWF), or 

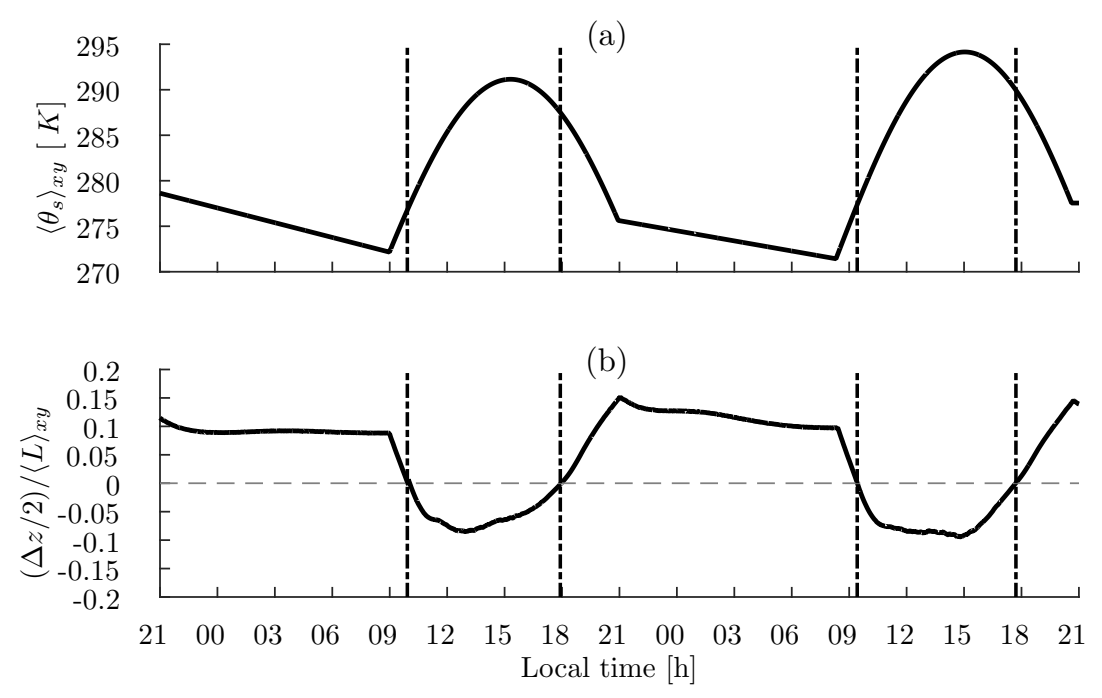

Figure 1: (a) Spatially averaged and time-dependent imposed potential temperature at the surface of the domain $\left\langle\theta_{s}\right\rangle_{x y}[K]$; (b) normalized stability parameter, $(\Delta z / 2) /\langle L\rangle_{x y}$, where $(\Delta z / 2)$ is the hight of the first grid-point and $L$ is the Monin-Obukhov length as a function of time.

equivalently seen as a single wind turbine. For all cases, the turbines were immersed within an atmospheric boundary layer that would evolve with time, such that the wind farms could experience different flow types with different atmospheric stratification. Precisely, each simulation was run for a total of 48 hours (real-time), in which the initial conditions and forcing of the flow was extracted from the CASES-99 field experiment. This means that the atmospheric flow evolved over two complete days. In order to ensure the simulation of a realistic diurnal cycle the numerical simulations were forced with a height-independent and timeconstant geostrophic wind together with a time-varying surface temperature. The values for the geostrophic wind and surface temperature were extracted from the CASES-99 field experiment between October 22-24, 1999. This period was previously studied by Kumar et al. [24] to illustrate the wellness of the LES simulations. Recently, Fitch et al. [41] and Sharma et al. [42] used the same period of time to investigate the effects of finite-size and very-large wind farms on a realistic diurnal cycle using mesoscale simulations (WRF) and LES, respectively. It is for this reason that the same forcing is used in this work.

The detailed time-evolution of the surface temperature is represented in Figure 1a, and the corresponding evolution of the atmospheric stratification is represented in Figure 1b, where the non-dimensional stability parameter $z /\langle L\rangle_{x y}$ is presented (with $z=\Delta z / 2$ ). The vertical dashed lines indicate the times with an atmospheric stratification change $(0955$, 1752, 0925 and 1842) and they will be used in other Figures throughout this work. In addition, the flow was forced with a geostrophic wind of value $\left(u_{G}, v_{G}\right)=(9,-3) \mathrm{ms}^{-1}$, and the domain size was set to $(2 \pi \times \pi \times 3) z_{i}$, where $z_{i}$ is the height of the boundary layer, initially located at $1000 \mathrm{~m}$ height. To keep a fairly high numerical resolution, a computational domain with $256 \times 128 \times 384$ grid points was used. The simulations where initialized with a height-independent geostrophic wind and a well mixed temperature profile matching the initial surface temperature of $278.6 \mathrm{~K}$, with an inversion layer spanning from $z_{i}$ to the top of 
(a)

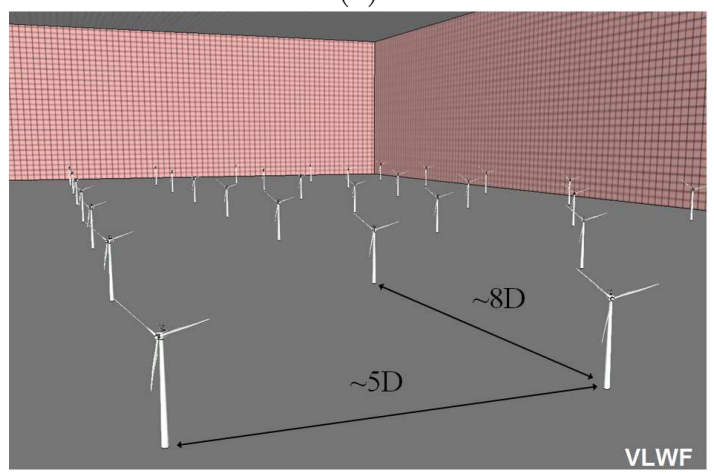

(b)

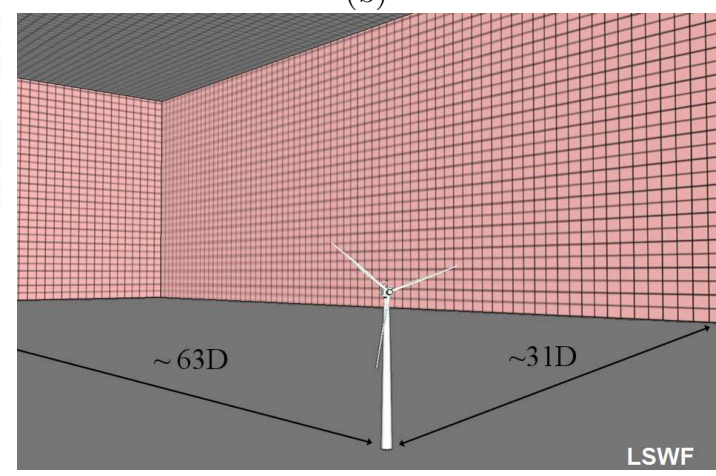

Figure 2: a) Very large wind farm case (VLWF); (b) Largely spaced wind farm case (LSWF).

the domain, with $z_{i}=1000 \mathrm{~m}$. The LES was initialized with vertical profiles of velocity and temperature extracted from Kumar et al. [25]. Overall, a total of 19 different cases (7 for VLWF and 12 for the LSWF) have been considered to evaluate the effect of the upstream scanning distances and orientation times.

\subsection{Very large wind farm (VLWF)}

The VLWF configuration consisted of 48 wind turbines homogeneously distributed in 8 rows and 6 columns using the traditional spacing of $\sim 8 D$ and $\sim 5 D$ (where $D$ refers to the turbines' rotor diameter) in the streamwise and spanwise directions, respectively (see Figure 2a for details). Similar to previous studies [43] the turbines' hub-height $\left(z_{h}\right)$ was located at $100 \mathrm{~m}$ and the wind turbine diameter was equally set to $100 \mathrm{~m}$. Besides parametrizing the wind turbines using the actuator disk (AD) with rotation approach of Wu et al.[30] including the dynamical yaw-alignement of Sharma et al.[26], the turbines were additionally capable of dynamically yaw-align with the incoming wind vector measured at different upstream scanning distances, similar to what a real wind turbine would do if equipped with a wind LIDAR. Therefore, each row of wind turbines was configured such to measure the incoming wind vector at a different upstream distance ranging between $50 \mathrm{~m}(D / 2)$ and $200 \mathrm{~m}(2 D)$ upstream $(D / 2,3 D / 4, D, 5 D / 4,3 D / 2$ and $2 D)$. In the most basic mode, the wind turbine model readjusts the yaw orientation every 10 minutes according to the averaged wind vector measured at the predetermined upstream scanning distance. The incoming wind vector is equally averaged for 10 minutes, and the angle of the incoming wind is measured as $\overline{\tan ^{-1}\left(\left\langle v_{d}\right\rangle /\left\langle u_{d}\right\rangle\right)}$, where the overline represents the time average and $\langle\cdot\rangle$ represents the spatial average of $u_{d}$ and $v_{d}$, which denote the horizontal velocities at the upstream disk area scanned by a hypothetical wind LIDAR. This configuration allowed us to study the effect of using different upstream scanning distances to measure the incoming wind vector and correspondingly readjust the turbine's yaw-alignment.

In addition, in this work we are interested in deciphering the effect of the averaging and readjustment time to determine the correct incoming wind vector, given a fixed upstream scanning distance (e.g. $D / 2$ ). For this reason, a suite of seven simulations was used, where each used a different averaging time to measure the upstream incoming wind vector (1 
(a)

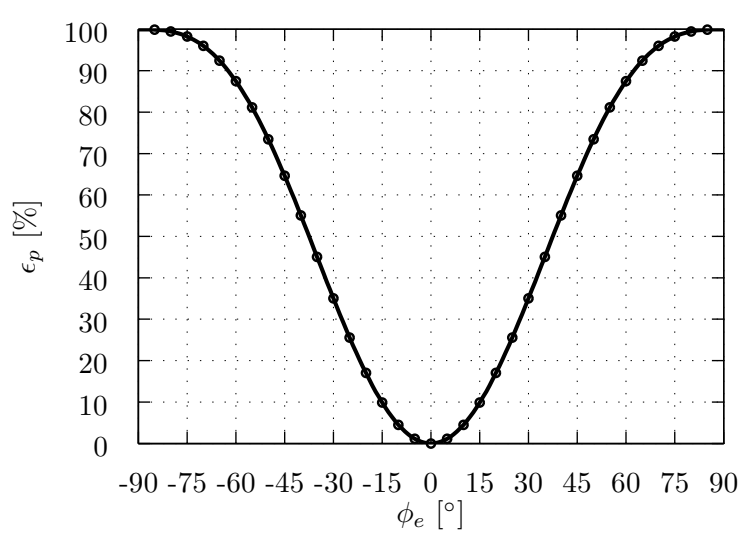

(b)

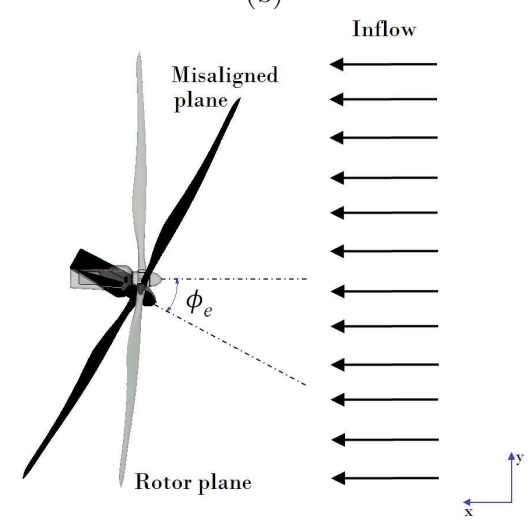

Figure 3: (a) Theoretical representation of the error in power $\left[\epsilon_{p}=\left(1-\cos ^{3}\left(\phi_{e}\right)\right) \times 100\right]$ due to the wind turbine misalignment; (b) schematic representation of the wind turbine misalignment.

second, 5 minutes, 10 minutes, 20 minutes, 30 minutes, 45 minutes, 60 minutes). Because of the unaffordable numerical cost it would imply running 7 additional diurnal cycles, each one considering a different averaging time, shorter simulations (4 hours of physical time) were performed for the day, unstable period (1330 to 1730), and for the night, stable period (0130 to 0530), so that the effect of stratification (unstable and stable) could be analyzed.

\subsection{Largely spaced wind farm (LSWF)}

The LSWF consists of a wind farm where the constituent wind turbines are highly spaced. This case could perfectly correspond to the case of a single wind turbine. Notice that given the size of the numerical domain, although it is periodic as mentioned in section 2, the wake of the wind turbine doesn't interact with itself, meaning that in practice we are effectively modeling a single wind turbine. For this case, the characteristics of the wind turbine are exactly the same as the ones described in the VLWF case. Equivalently, the turbines forming this very large spaced wind farm would be in practice separated by $\sim 63 D$ and $\sim 31 D$ in the streamwise and spanwise directions, respectively (see Figure $2 \mathrm{~b}$ for details). In this case, and as a result of the computational cost of the simulations, only 4 upstream scanning configurations were considered $(D / 2, D, 3 D / 4$, and $2 D)$. Note that for each upstream scanning configuration 48 hours of real time flow were simulated. Similar to what was done in the VLWF configuration, the same shorter events, representing unstable and stable stratified conditions, were used to study the effect of the different averaging times (1 second, 5 minutes, 10 minutes, 20 minutes, 30 minutes, 45 minutes, 60 minutes).

\section{Results}

The nominal loss in harvested power induced by a given yaw-misalignment angle is provided by the cosine cubed of the misalignment angle $\left(\cos ^{3} \phi_{e}\right)$. It is for this reason that the turbine's harvested power is highly sensitive to the turbine's capacity to timely align with the incoming wind vector. In Figure 3b, a graphical representation of the yaw-misalignement 
Table 1: Parameters used to estimate the economical loss for two given average yearly misalignments and for two distinct scenarios, Horns Rev 2 in current operational conditions and for the projected Horns Rev 3 wind farm

\begin{tabular}{|l|l|l|}
\hline & Horns Rev 2 & Horns Rev 3 (Projected) \\
\hline Number of wind turbines & 91 & 50 \\
\hline Wind farm nominal power & $209 \mathrm{MW}$ & $400 \mathrm{MW}$ \\
\hline Capacity factor (CF) & $50 \%$ & $65 \%$ \\
\hline Estimated annual energy production & $915 \mathrm{GWh}$ & $2,278 \mathrm{GWh}$ \\
\hline Bid price & $25 € / \mathrm{MWh}$ & $103 € / \mathrm{MWh}$ \\
\hline Yearly income & $13 \mathrm{M} €$ & $235 \mathrm{M} €$ \\
\hline Revenue loss for a misalignment of $5^{\circ}$ & $0.3 \mathrm{M} € /$ year & $3.1 \mathrm{M} € /$ year \\
\hline Revenue loss for a misalignment of $10^{\circ}$ & $1.1 \mathrm{M} € /$ year & $10.5 \mathrm{M} € /$ year \\
\hline
\end{tabular}

of a wind turbine with the incoming wind vector is presented. Figure 3a indicates the corresponding loss in power induced by different degrees of misalignment. It can be observed that yaw errors of $10^{\circ}$ can reduce the harvested power by about $5 \%$, and errors of $15^{\circ}$ can reduce as much as $10 \%$ of the harvested power. While these values might seem small a priori, their corresponding economical translation is certainly important. In Table 1 it is shown an estimation of the economical loss in revenue due the wind turbine misalignment. The analysis is performed for two different offshore large wind farm scenarios: the well known Danish Horns Rev 2 wind farm and the projected Horns Rev 3, located at the North Sea. Results show that for a misalignment of $5^{\circ}$ the total economical loss is $0.3 \mathrm{M} € /$ year and $3.1 \mathrm{M} € /$ year for the Horns Rev 2 wind farm and Horns Rev 3, respectively. Additionally, for a misalignment of $10^{\circ}$ it corresponds to a loss of $1.1 \mathrm{M} € /$ year and $10.5 \mathrm{M} € /$ year. The data to compute the economics has been extracted from the Danish energy agency [44] assuming a capacity factor of $65 \%$ for the Horns Rev 3 and an average bid price of $25 € / \mathrm{MWh}$ for the Horns Rev 2, representative of the 2014-15 energy market [45, 46]. The estimated annual energy production of a wind farm is obtained by multiplying the nominal power of the wind farm by a total year time and the corresponding wind farm capacity factor. By multiplying the energy bid price by the estimated annual production, one obtains the yearly income. To obtain the revenue loss, the yearly income should be multiplied by the error in harvested power due to the yaw-misalignment of the turbine depicted in Figure 3b. Overall, the loss in income showed in Table 1 partially illustrates the urgent need for reducing the misalignment error.

\subsection{Optimal upstream distance at which wind LIDARs should interrogate the atmosphere}

One of the objectives of this work is to assess whether there exists an optimal upstream scanning distance for wind LIDARs to retrieve the most-accurate yaw-alignment angle considering the effect of changing atmospheric stratification. At the same time, we aim to better analyze the potential yaw-misalignment induced by measuring with a wind vane placed behind the rotor blades.

Subplots $a_{i}$ and $b_{i}$ in Figure 4 present vertical profiles of wind velocity together with their corresponding incidence angle for a 10-minute period representative of the unstable 
stratification (i.e. averaged between 0230 and $0240 \mathrm{pm} ; i=1$ to 3 ) and for both cases, the LSWF $\left(4 a_{i}\right)$ and the VLWF $\left(4 b_{i}\right)$. Subplots $4 c_{i}$ and $4 d_{i}$ illustrate the same profiles for a 10-minute period representative of the stable stratification (i.e. averaged between 0030 and 0040am) also for both cases the LSWF $\left(4 c_{i}\right)$ and the VLWF $\left(4 d_{i}\right)$. The vertical profiles have been planar-averaged (denoted by the sub-index $y$ ) along the upstream plane parallel to the turbine's rotor disk, and the vertical axis is normalized with the turbines' hub-height $\left(z_{h}=100 \mathrm{~m}\right)$. Subplots $4 a_{1}-d_{1}$ represent the magnitude of the incoming wind $\left(\bar{U}=\sqrt{\bar{u}^{2}+\bar{v}^{2}+\bar{w}^{2}}\right)$ at different upstream distances $(D / 2,3 D / 4, D, 5 D / 4,3 D / 2,2 D)$ and at the traditional nacelle's wind vane location $(-3 D / 10)$ normalized by the geostrophic wind $\left(\bar{U}_{G}=\sqrt{\bar{u}_{G}^{2}+\bar{v}_{G}^{2}}\right)$. For all the subplots, the turbine's rotor-disk region is constrained between two horizontal dot-dashed lines, and the precise location of the hub is indicated with a horizontal dash line. Because the differences in wind magnitude are small between all the upstream scanning distances the relative differences with respect to the measured wind magnitude at $\left.D / 2\left(\left\langle\bar{U}_{i}\right\rangle_{y}-\left\langle\bar{U}_{D / 2}\right\rangle_{y}\right) /\left\langle\bar{U}_{D / 2}\right\rangle_{y}\right)$ are also presented in subplots $4 a_{2}-d_{2}$. The $D / 2$ distance has been selected as a reference upstream measure because it is close to the wind turbine and still outside of the induction zone [47]. Finally, subplots $4 a_{3}-d_{3}$ represent the normalized relative difference with the yaw-angle measured at $D / 2,\left(\left\langle\bar{\phi}_{i}\right\rangle_{y}-\left\langle\bar{\phi}_{D / 2}\right\rangle_{y}\right) /\left\langle\bar{\phi}_{D / 2}\right\rangle_{y}$.

In the measure of the incoming wind, we observe a maximum difference of $7.4 \%$ between the furthest upstream scanning distance $(2 D)$ and the wind vector at $D / 2$ for the LSWF and a difference of $4.6 \%$ for the VLWF, both during the unstable stratification period (subplots $4 a_{2}$ and $4 b_{2}$ ). For the closer upstream scanning distances these differences decrease. As one would expect, the measurements from the wind vane proxy present larger differences with a maximum difference of $20.2 \%$ and $16 \%$ for the LSWF and the VLWF cases, respectively. During the stably stratified period (subplots $4 c_{2}$ and $4 d_{2}$ ) maximum differences of $6.7 \%$ and $4.7 \%$ between the furthest upstream scanning distance $(2 D)$ and the wind vector at $D / 2$ are observed for the LSWF the VLWF case, respectively. Also, the measurements for the wind vane proxy present larger differences with a maximum of $17.3 \%$ for the LSWF and $13 \%$ for VLWF case. The average standard deviations of the relative velocities at hub height are $0.43 \%$ and $1.5 \%$ correspondingly for the VLWF under unstable and stable conditions, and $4 \%$ and $2 \%$ for the LSWF for the unstable and stable cases.

In the measure of the yaw-angle, the most important is to note the quasi-inexistent difference obtained when measuring at different upstream distances, regardless of the stratification. Once more, it is interesting to notice the large differences with the measurement position behind the blades. Once the flow crosses the rotor disk area, there exists a large deflection angle of up to $22.4 \%$ for the LSWF and close to $9 \%$ for the VLWF for the different stratifications. Similarly, the measured yaw-angle for the stable period (see subplots $4 c_{3}$ and $4 d_{3}$ ) remains practically the same for all the upstream scanning distances, and the nacelle angle is deflected by smaller values in comparison to the unstable regime. Note that the characteristic difference in magnitude between the results obtained for the VLWF and the LSWF is a result of the higher turbulence intensity in the VLWF that tends to homogenize the turbulent flow around the wind turbines $[48,49,50]$. Also, the proxy for the incoming wind vector measured by the wind vane corresponds to an approximate measure a wind vane would provide when installed at the turbine's nacelle, right behind the rotor-blades 

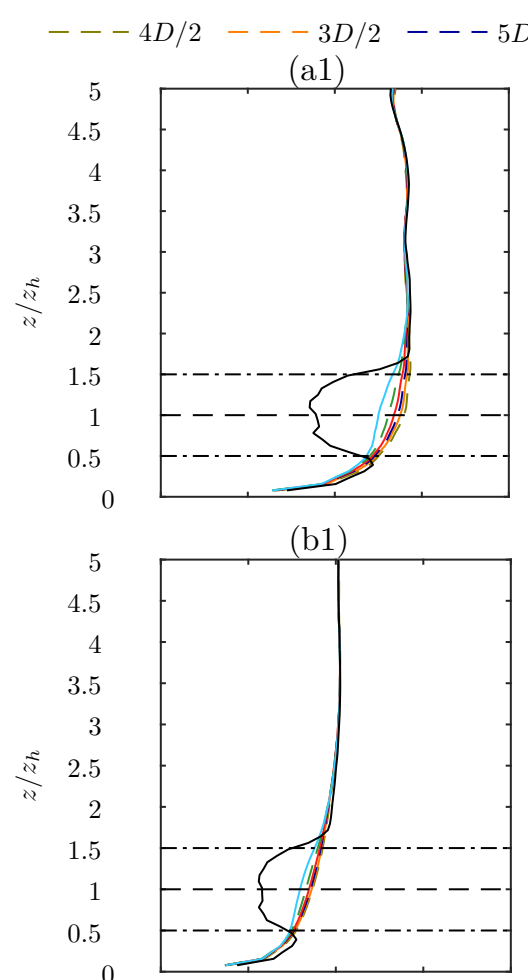

(c1)

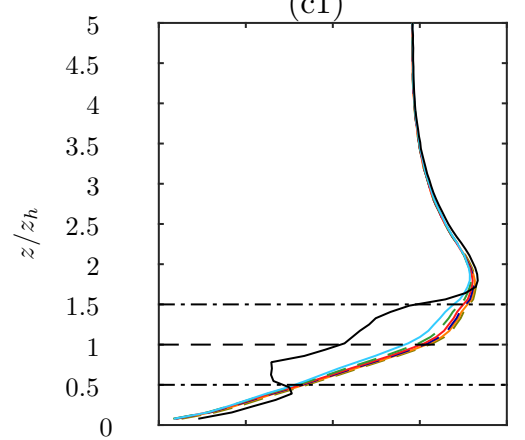

(d1)

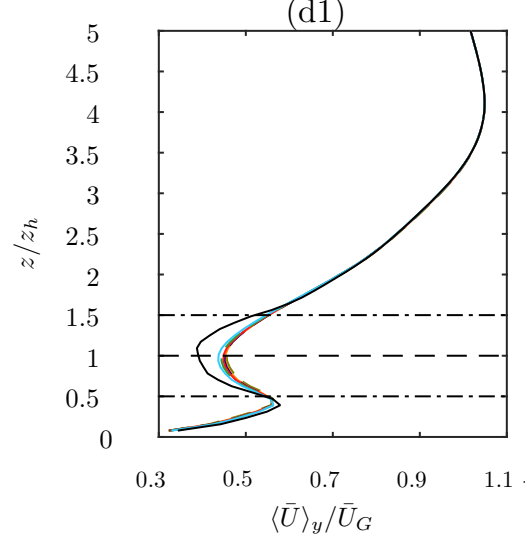

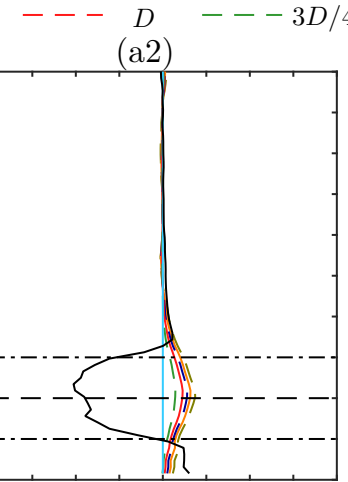

(b2)

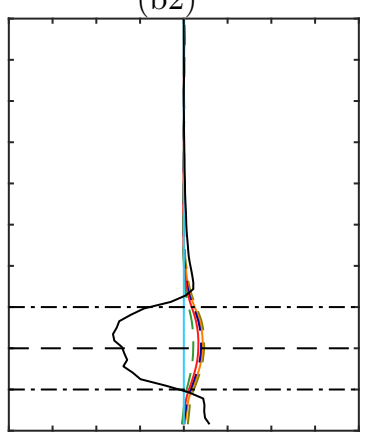

(c2)

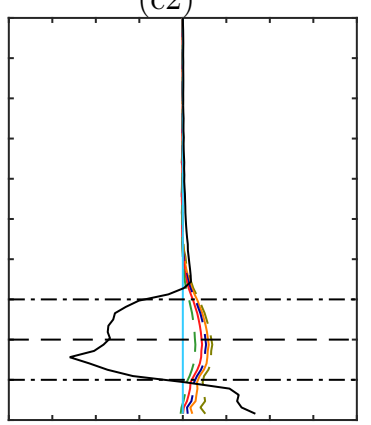

(d2)

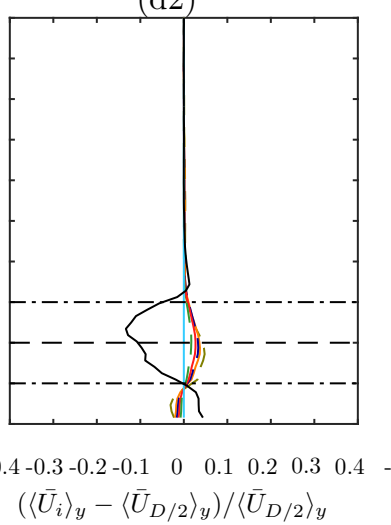

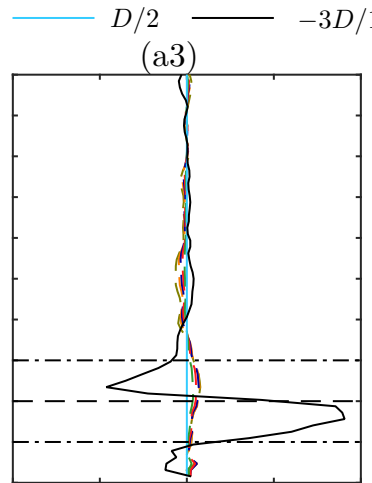

(b3)

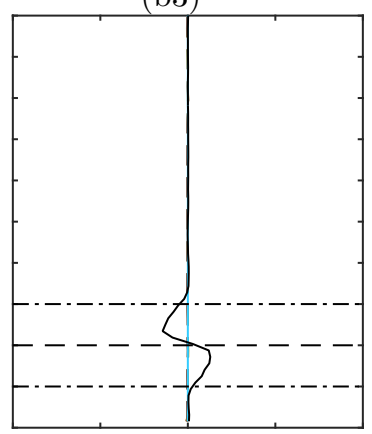

(c3)

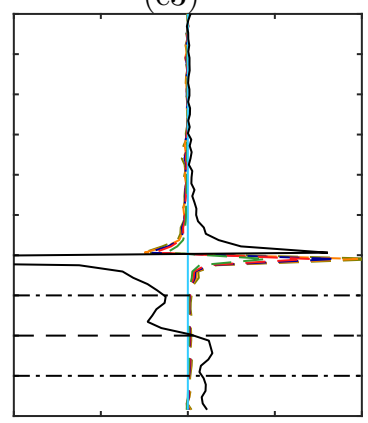

(d3)

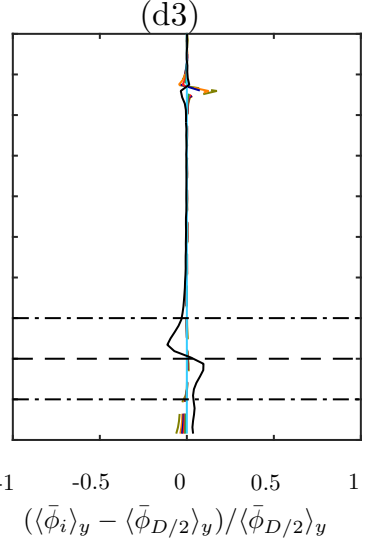

Figure 4: Vertical averaged profiles of normalized velocity magnitude $\left(a_{1}\right.$ and $\left.c_{1}\right)$, relative velocity magnitude $\left(a_{2}\right.$ and $\left.c_{2}\right)$ and relative angle $\left(a_{3}\right.$ and $\left.c_{3}\right)$ for the LSWF for the stable $\left(a_{i}\right)$ and unstable $\left(c_{i}\right)$ stratified periods. A similar structure is used in subplots $b_{i}$ and $d_{i}$ for the VLWF case, respectively. 
and without being corrected with the often used NTFs.

By comparing subplots $\left(4 a_{i}\right.$ and $\left.b_{i}\right)$ to subplots $\left(4 c_{i}\right.$ and $\left.d_{i}\right)$ the effect of atmospheric stratification on the incoming wind vector (module and angle) is clearly observed. At night, during the stable period, a characteristic nocturnal low-level jet (LLJ) forms at heights between $1 \leq z / z_{h} \leq 2$, which interacts with the top section of the wind turbine blades. In the case of a VLWF the LLJ is shifted by the presence of the very large wind farm [51]. While the LLJ induces relevant changes on the wind vector profiles, the relative differences between the different upstream scanning regions remain similar between the day-time and night-time.

Overall, the results presented within Figure 4 show that there are no significant differences in the incoming wind vector when measured at different upstream distances and for different atmospheric stability conditions. Even thought Figure 4 shows the behavior of 10 minutes data during the unstable-stratified and stable-stratified periods, a similar behavior is observed for different 10 minutes averaged periods along the two diurnal cycles (not shown here for the sake of clarity). This result is really important because it will allow the nacelle installed wind LIDARs to learn from the incoming wind vector at further upstream distances, providing more time to the wind turbines to readjust their corresponding settings and hence optimally adapting to the incoming flow. Results are the opposite for the nacelle wind vane location. In this case, results show how the measured flow diverges from the one reaching the blades, with errors of up to $22.41 \%$ in the measure of the wind vector angle. These results are in-line with experimental measurements done using a real wind LIDAR mounted on the nacelle of a wind turbine [7]. Currently, wind turbine operators get around these large errors by imposing an 'ad-hoc' correction function using an unperturbed wind vector measurement from a meteorological-tower, upstream of the farm. Yet, these correction functions rarely account for the effect of atmospheric stability, which is shown to have a relevant effect in the deflection of the yaw-angle measured at the nacelle for the LSWF case (30\% and $20 \%$ for the unstable and stable in average, respectively), and a more attenuated effect for the VLWF case (10\% and 5\% for the unstable and stable in average, respectively), right behind the blades. The reduced wind-angle deflection on the VLWF is a result of the enhanced mixing produced by the large array of wind turbines.

To further explore the effect of atmospheric stratification on the incoming wind vector, Figure 5 illustrates the average yaw angle $\left(\langle\bar{\phi}\rangle_{W T}\right)$ as a function of the local time for a duration of two complete diurnal cycles, where $\left(\langle\cdot\rangle_{W T}\right)$ represents the average among the distinct wind turbines. The yaw angle has been spatially averaged on the projection of the rotor disk at the different upstream distances, and therefore it is referred as an upstream rotor-disk average. Within this Figure, subplot 5a represents the average evolution of the yaw angle in the case of a LSWF and the blue shade indicates the corresponding standard deviation for the four different upstream scanning distances presented in section 3.2. Supblot $5 \mathrm{~b}$ depicts the case of the VLWF, where the blue line represents again the wind vector orientation averaged over all the wind turbines conforming the wind farm, and the blue shading indicates the corresponding standard deviation. The reason for averaging over all the wind turbines, without distinguishing between the turbines scanning at different upstream distances, is the similitude presented in Figure 4. Further, the black line represents the 

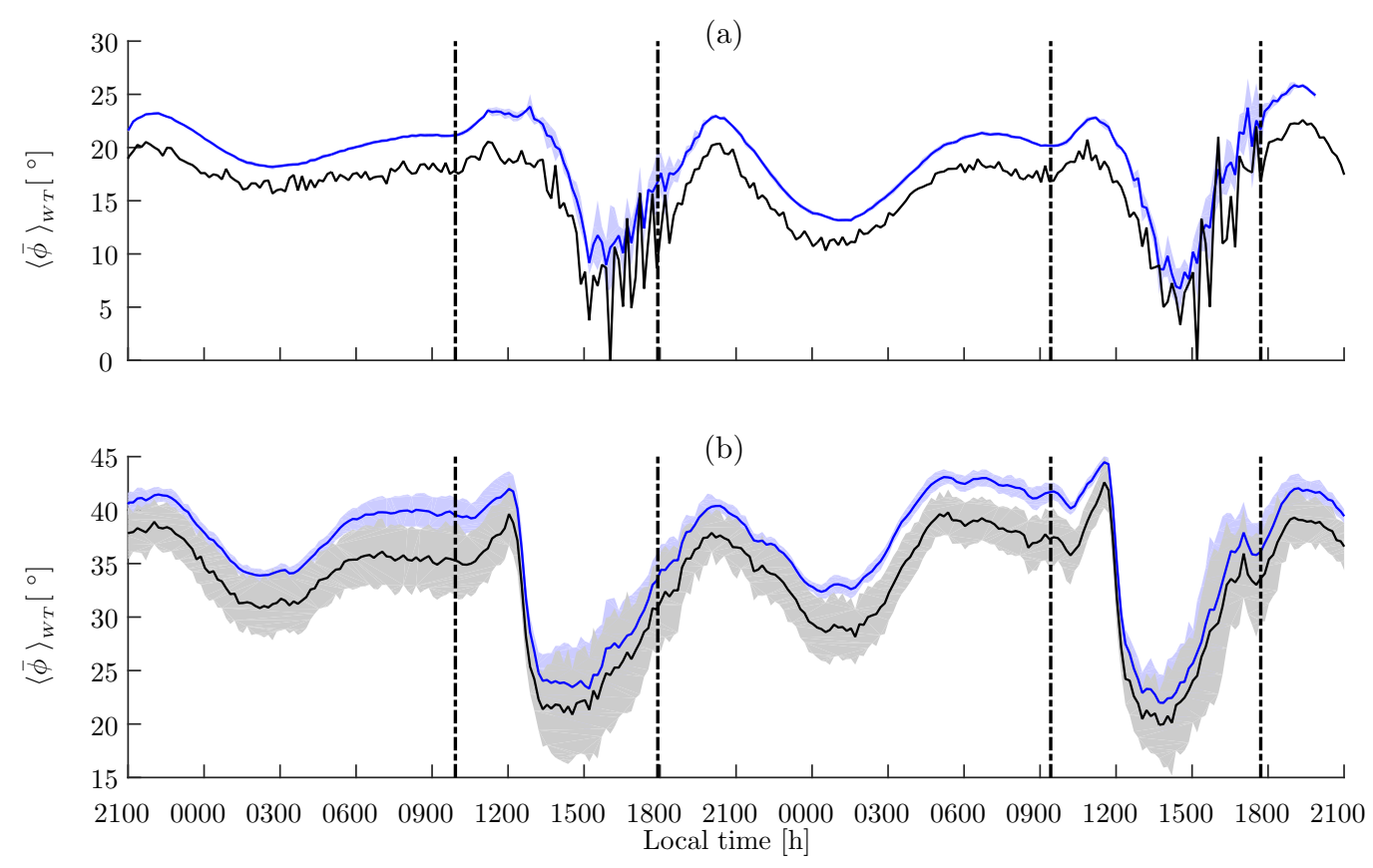

Figure 5: Yaw alignment for the upstream scanning distance (in blue) and for the wind vane (in black), (a) for the LSWF and (b) for the VLWF.

average wind vector angle obtained by those wind turbines that learn about the incoming wind measuring behind the blades, as a proxy to a wind vane and cup anemometer, and the black shade represents the corresponding standard deviation. It is interesting to note that during the unstable regimes, as a result of the enhanced vertical and lateral mixing $[52,53,54,55]$ there exists a larger variability (standard deviation) in the wind vector angle measured among the different turbines in comparison to the stably stratified regime. However, this differentiated behavior is not apparent in the wind turbines driven by the wind vane proxy, probably as a result of the continuous mixing produced by the blades regardless of the atmospheric stratification. Interestingly, the maximum error observed between the upstream and wind vane proxy measurements are close to $5^{\circ}$ during the stable regime (from 0600 to 0900 in the first diurnal cycle). Almost no differences are observed during the unstable regime (from 1230 to 0100 of the first diurnal cycle). In subplot 5a, the same representation is used, where the maximum error is close to $4^{\circ}$ and the minimum is close to $1^{\circ}$. Mikkelsen et al. [7] showed experimental misalignments of $10^{\circ}$ root mean square (RMS) value for a 10 minutes average of a 3 hours 20 minutes observation period during the night of April 30th, 2009. This is about half the error observed in subplot 5a. This underprediction of the LES could be due to the approximate wind turbine model used in the LES to represent the actual turbines. It is also interesting to note the difference in averaged angle values between the VLWF and LSWF cases. This is a result of the enhanced resistance introduced by the large wind farm in comparison to the largely spaced wind farm, which produces a deeper geostrophic imbalance.

As a result of the diurnal evolution of the yaw-angle and its associated standard deviation, 
a new unknown arises with respect to the use of wind LIDAR technology. From the presented results, it is clear that the scanning averaging time and corresponding turbine's readjustment frequency should also be analyzed. For example, one could wonder whether it could be more optimal to keep the wind turbine's yaw alignment fixed during day time, when the wind vector continuously changes direction and just allow the time-varying readjustment during night-time, when the flow changes less randomly. This additional degree of freedom is explored in the following section.

\subsection{Optimal averaging time to interrogate the atmosphere}

A total of 14 simulations of 4 hours real-time length each have been performed to evaluate the effect of using different averaging times to readjust the turbine's yaw-alignment. The study includes both (stable and unstable) stability regimes for the two wind farm scenarios (VLWF and LSWF). In this case, the analysis is based on the same previous diurnal cycle, but only consider the time period between 0130 and 0530 to represent the stable regime, and the time period between 1330 and 1730 to represent the unstable stratification. During these periods of time the wind turbine model was configured as such to learn from the incoming wind vector at a fixed distance upstream of the rotor disk $(D / 2)$. The corresponding frequency of yaw-readjustment was varied, with time-periods ranging between 1 second and 60 minutes. Precisely, the studied readjustment time-periods are: 1 second, 5 minutes, 10 minutes, 20 minutes, 30 minutes, 45 minutes, and 60 minutes, where each set-up corresponds to a new numerical simulation in which all turbines are equally configured. These times also correspond to the averaging times used to determine the characteristics of the incoming wind vector (module and angle). In order to quantify the misalignment error with the incoming wind vector and hence the corresponding loss in power induced by the use of different averaging times, the simulation with one-second readjustment-time has been used as a base case, or 'ground truth'.

In order to evaluate the performance of the different yawing times, Figure 6 illustrates the normal probability density function (PDF) of the yawing error associated with the different study cases (VLWF versus LSWF, day-night for different averaging times). The error is computed as the difference between the angle of the incoming wind vector measured with a $1 \mathrm{~Hz}$ frequency and the angle obtained by the corresponding longer averages i.e. $\epsilon_{\phi}=\bar{\phi}^{1 s}-\bar{\phi}^{t}$, where $t$ represents the different averaging times. Subplots $6 \mathrm{a}$ and $6 \mathrm{~b}$ represent the study cases for the LSWF and subplots $6 \mathrm{c}$ and $6 \mathrm{~d}$ for the VLWF, where $6 \mathrm{a}$ and $6 \mathrm{c}$ represent the stably stratified case and $6 \mathrm{~b}$ and $6 \mathrm{~d}$ illustrate the unstable case, respectively. Notice that the left and right dashed lines represent the $95 \%$ confidence intervals, which are equivalent to two standard deviations $(2 \sigma)$, for the 30 minutes PDF. It is worth noting that the unstable regimes are characterized by a wider PDF, which is related to the variability of the wind vector during the unstable regime. Subplot 6 a shows that the probability density function of the associated error for the LSWF during the stably stratified period is overall quite small (less than $1^{\circ}$ on the 60 minutes period case and for the mean value) when compared to the other scenarios, regardless of the averaging time. This means that even if one decides to use large periods of time between yaw-readjustments, the associated penalization in power loss would be very small. Also, it is interesting to notice that all the PDFs in subplot 6a are 

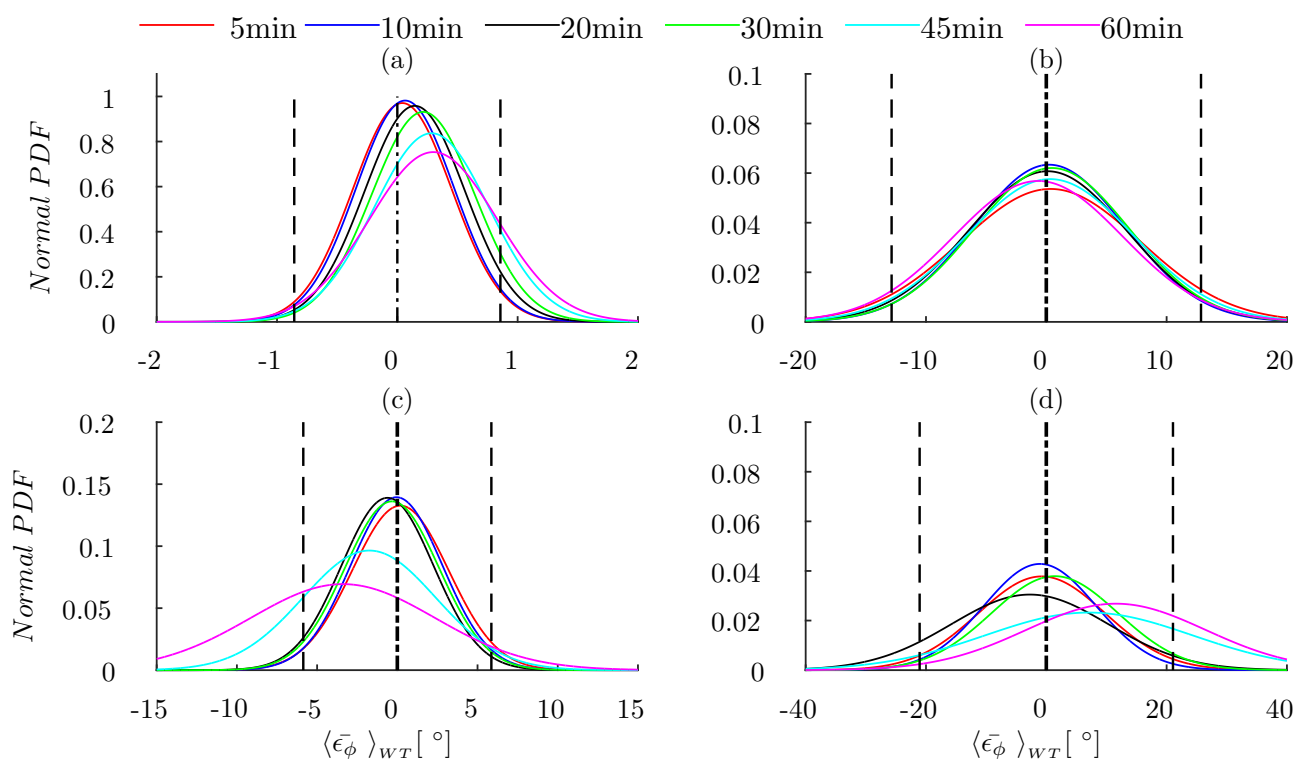

Figure 6: Probability density function of the yaw-alignment error for the LSWF during the stable (a) and unstable (b) regimes, and for the VLWF, (c) and (d), respectively.

shifted towards the right. This phenomena is an effect of the preferential wind direction. For example, if the wind vector has a preferred clockwise rotation in time, the increase of the turbine's yawing time leads to larger errors. This is the result of the cumulative misalignment with longer times. This behavior is not seen in subplot $6 \mathrm{c}$ due to the high perturbation produced by the presence of a large wind farm. Also, in subplots $6 \mathrm{~b}$ and $6 \mathrm{~d}$ it is not observed as a result of the large mixing and not preferred direction of the flow during the unstable period.

In contrast, in the convective period for the LSWF case (subplot 6b), the means of the PDFs associated with the different readjustment periods are progressively shifted to larger biased errors with $95 \%$ confidence of $\pm 12.86^{\circ}$ contrasting with a $\pm 0.86^{\circ}$ for the stable period. Also, it is noticeable the larger standard deviation associated in this case, which is more than ten times larger than the one for the stable stratified period. In the VLWF cases (subplot $6 \mathrm{c}$ and $6 \mathrm{~d}$ ) results follow a similar trend with larger standard deviations on the unstable stratified period. However, in this case the error associated with larger readjustment periods is much more noticeable, with PDF mean values in exceedance of $5^{\circ}$ during the stable stratified period, and larger than $10^{\circ}$ during the unstable stratified period. Therefore, in VLWFs the continuous interaction between wakes truly penalizes the large yawing times with large errors observed, both during day-time and night-time (subplots $6 \mathrm{c}$ and $6 \mathrm{~d}$, respectively).

A better representation of the relationship between the yawing angle error presented in Figure 6 and its direct effect on the harvested power is presented in Figure 7. This figure illustrates the normalized cumulative error in power for the different atmospheric stability scenarios and for a period of time of 150 minutes. The associated error in harvested power $\left(\epsilon_{P}\right)$ is computed using the theoretical $\cos ^{3}$ factor, provided the error in the yaw angle $\left(\epsilon_{\phi}\right)$, 

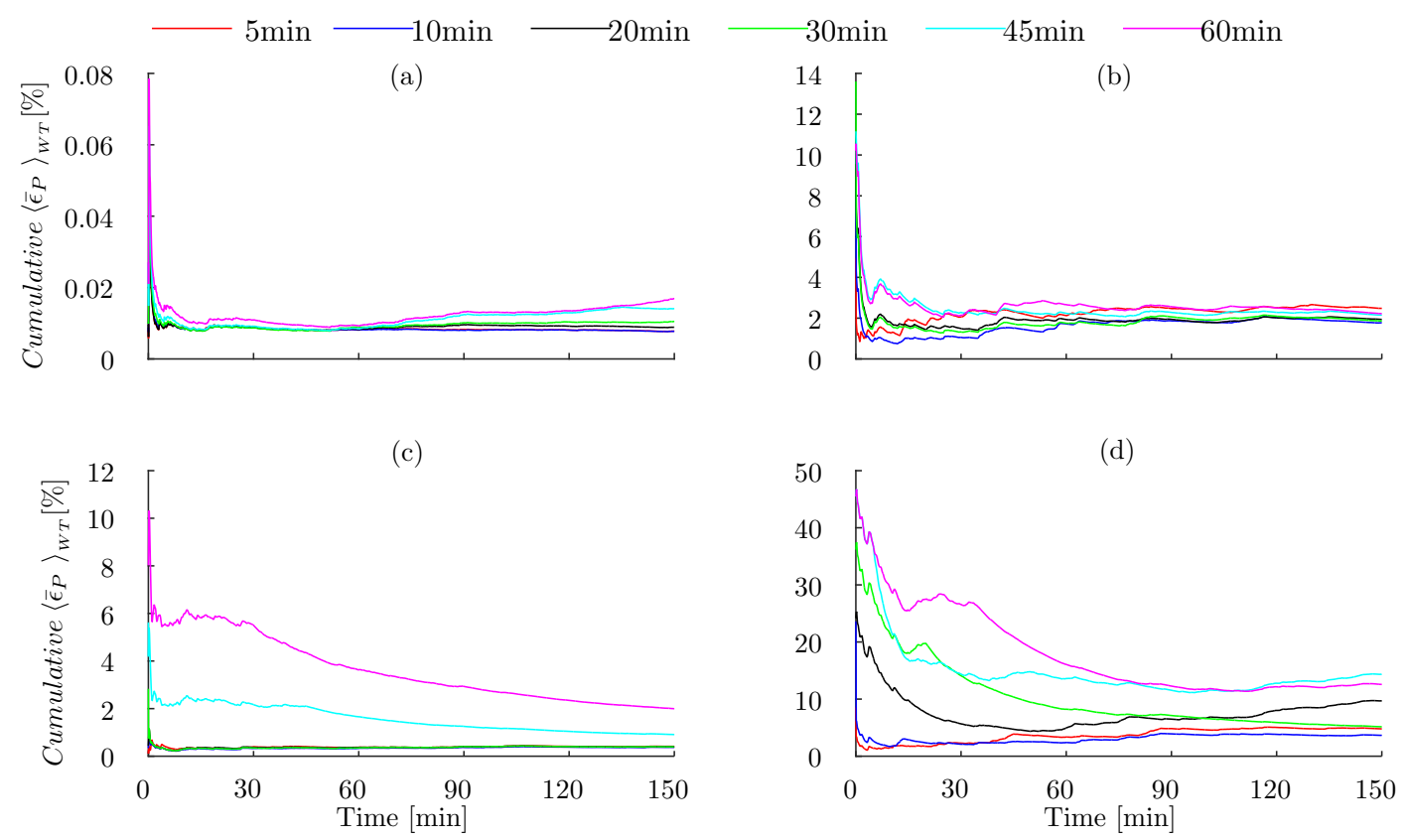

Figure 7: Normalized cumulative error in power for the LSWF during the stable (a) and unstable (b) regimes, and for the VLWF, (c) and (d), respectively.

meaning

$$
\left\langle\bar{\epsilon}_{P}\right\rangle_{W T}[\%]=\frac{100}{N} \sum_{t=1}^{N}\left(1-\left|\cos ^{3}\left(\left\langle\bar{\epsilon}_{\phi}\right\rangle_{W T}\right)\right|\right),
$$

where $\mathrm{N}$ is the time cumulative number of events used to compute the error with a frequency of $1 \mathrm{~Hz}$ over 150 minutes $(\mathrm{N}=9000)$ and the error in the yaw angle is computed as $\left\langle\bar{\epsilon}_{\phi}\right\rangle_{W T}=$ $\left\langle\bar{\phi}^{1 s}-\bar{\phi}^{t}\right\rangle_{W T}$, where $t$ represents the different averaging times. Notice that the initial cumulative error values ( $\leq$ than the first 60 minutes) have a poor statistical meaning given the reduced amount of cumulative data points. The cumulative error becomes representative after the 90 minutes threshold (where $\mathrm{N}$ is equal to 5400). This explains the very large error encountered during the initial 5-10 minutes of the analysis, where $\mathrm{N}$ is still very small (300600 data points respectively).

Interestingly, during the stable period for the LSWF (subplot $a$ ) the cumulative error in power is practically negligible (less than $0.02 \%$ ), showing the excellent performance of the upstream scanning approach regardless of the yawing times. Nonetheless, it can be observed that the 5, 10 and 20 minutes readjustment times perform the best, as one could a priori expect. The small error associated to this case is due to the fact that the incoming wind is not perturbed by other wind turbines as well as due to the strong incoming winds associated with the LLJ. For the unstable stratified scenario of the LSWF (subplot 7b) there is a slight increase of the cumulative error in power as a result of the unstable ABL, but once again it presents small differences between the different readjustment times. Interestingly, the 10, 20 and 30 minutes readjustment periods present the lowest associated error in power. 
Indeed, it is the 5 minute readjustment time that presents the largest cumulative error. Results show how the strong fluctuating winds characteristic of the day-time periods penalize the shorter readjustment time. It has been observed that once the yaw alignment has been corrected, it doesn't take long for the wind vector to change its orientation, inducing continuous large misalignment. In contrast, while larger times such as the 30 minutes readjustment period remains most of the time aligned, the absolute misalignment error remains smaller in magnitude.

Finally, subplots $7 \mathrm{c}$ and $7 \mathrm{~d}$ represent the cumulative error in power for the VLWF. In both cases (stable and unstable regimes) results illustrate the effect of the continuous wake interaction, characteristic of large wind farms [52, 53, 54, 55], with much larger errors for the longer readjustment times. A $2 \%$ and $12.5 \%$ cumulative error is observed for the 60 minutes readjustment-time for the stable and unstable cases respectively. Also in this case, the error appears to be larger for the convective time in comparison to the stable period.

Overall, the 30 minutes readjustment period seems to perform optimally within all the studied scenarios, presenting a small error for all the different cases independently of the stability and the particular wind farm scenario.

\section{Conclusions}

The goal of the present study was to investigate the possible existence of an optimal upstream scanning distance and readjustment-time to accurately learn about the incoming wind vector using wind LIDARs mounted on the wind turbine nacelle. To answer this question a total of nineteen LES cases have been developed which consider the canonical scenarios of a largely spaced wind farm (LSWF) and a very large wind farm (VLWF) under continuously changing atmospheric conditions.

The numerical results of two consecutive diurnal cycles reveal that during unstable regimes, as a result of the enhanced vertical and lateral mixing, there exists a larger variation in the incoming wind vector angle $\left(\sim 8^{\circ}\right)$ compared to the stable period, which shows a $50 \%$ reduction in wind angle variability. For the LSWF under stable stratification the maximum error observed between the upstream and wind vane proxy measurements of wind direction are close to $5^{\circ}$ (found between $0600 \mathrm{~h}$ and 0900h) whereas almost no differences were observed during the late unstable period (between $1300 \mathrm{~h}$ and $1800 \mathrm{~h}$ ). For the VLWF case, the maximum error is close to $4^{\circ}$ and the minimum is close to $1^{\circ}$. These results agree well with the experimental data of Mikkelsen et al. [7]. Nonetheless, some differences exist, which have been attributed to the approximate wind turbine model used in the LES to represent the wind turbines. However, results reveal no significant differences when measuring the incoming wind vector at different upstream distances, regardless of the atmospheric stratification. This is a very important result because it will allow wind LIDARs to learn from the incoming wind vector at far upstream distances, hence providing longer readjustment times to reset the optimal turbine parameters.

Within this study the effect of the wind turbine yaw readjustment-time has also been considered. Results indicate that for the LSWF under stable conditions the overall cumulative error in power is very small $(\sim 0.015 \%)$, with time periods of 5,10 and 20 minutes 
showing the best performance, and for the unstable conditions the 10, 20 and 30 minutes readjustment times showing the best performance ( $\sim 2 \%$ error $)$. In this case the 5 minutes period under-performed as a result of the fast and continuous change in the incoming wind vector ( $\sim 3 \%$ error). In contrast, for the VLWF (cases, both stable and unstable) illustrate the effect of the continuous turbine-wake interactions, with much larger errors for longer readjustment times. For example, a $2 \%$ and $12.5 \%$ cumulative error in power are observed for the 60 minutes readjustment time for the stable and unstable atmospheric regimes correspondingly. Overall, the 30 minutes readjustment period seems to perform most optimally, presenting small errors for all the different study cases, independently of atmospheric stability and the particular wind farm configuration.

\section{Acknowledgements}

This research has been supported with the start-up funds provided by the Mechanical Engineering Department at University of Utah to Assistant Professor Marc Calaf. The authors would like to acknowledge the computational support provided by the Center for High Performance Computing (CHPC) at University of Utah. PhD student Varun Sharma thanks the support received through the Swiss National Science Foundation (project no. 200021134892/1 and 20020 125092), ETH Domain Centre for Competence in Environmental Sustainability, and the NSERC Discovery Grant (MBP) Scientific IT and Application Support (SCITAS) group at EPFL. Finally, we wish to acknowledge the editorial assistance of James Kris Koford PhD, Department of Writing and Rhetoric studies, University of Utah.

\section{References}

[1] N. Goudarzi, W. D. Zhu, A Review of the Development of Wind Turbine Generators Across the World 4 (PARTS A AND B) (2013) 192-202.

[2] R. Wiser, M. Bolinger, 2014 Wind Technologies Market Report, Tech. Rep. August, U.S. Department of Energy (2015).

[3] M. Gryning, H. H. Niemann, M. P. S. Gryning, Q. Wu, M. Blanke, S. Member, Wind turbine inverter robust loop-shaping control subject to grid interaction effects, IEEE Transactions on sustainable energy 7 (1) (2016) 41-50.

[4] S. Wan, L. Cheng, X. Sheng, Effects of yaw error on wind turbine running characteristics based on the equivalent wind speed model, Energies 8 (7) (2015) 6286-6301.

[5] D. Shin, H. Kim, K. Ko, Analysis of wind turbine degradation via the nacelle transfer function, Journal of Mechanical Science and Technology 29 (9) (2015) 4003-4010.

[6] B. J. Vanderwende, J. K. Lundquist, The modification of wind turbine performance by statistically distinct atmospheric regimes, Environmental Research Letters 7 (3) (2012) 1-7.

[7] T. Mikkelsen, N. Angelou, K. Hansen, M. Sjöholm, M. Harris, C. Slinger, P. Hadley, R. Scullion, G. Ellis, G. Vives, A spinner-integrated wind lidar for enhanced wind turbine control, Wind Energy 16 (2013) 625-643.

[8] Benedetti-Michelangeli, G. Congeduti, G. Fiocco, Measurement of aerosol motion and wind velocity in the lower troposphere by doppler optical radar, Jouranal of Atmospheric Sciences 29 (1972) 906-910.

[9] Benedetti-Michelangeli, G. Congeduti, G. Fiocco, Determination of vertical eddy diffusion parameters by doppler optical radar, Atmospheric Environment 8 (1974) 793-799.

[10] E. Eloranta, J. King, J. Weinman, The determination of wind speeds in the boundary layer by monostatic lidar, Journal of Applied Meteorology 14 (1975) 1485-1489. 
[11] G. Iungo, W.Yu-Ting, F. Porté-Agel, Field measurements of wind turbine wakes with lidars, Jouranl of Atmospheric and Oceanic Technology 30 (2) (2012) 274-287.

[12] M. Harris, D. Bryce, A. Coffey, D. Smith, J. Birkemeyer, U. Knopf, Advance measurements of gusts by laser anemometry, Wind Engineering and Industrial Aerodynamics 95 (2007) 1637-1647.

[13] E. Simley, L. Pao, R. Frehlich, B. Jonkman, N. Kelley, Analysis of wind speed measurements using continuous wave lidar for wind turbine control, in: A. I. of Aeronautics, A. Astronautics (Eds.), Proceedings of 49th AIAA Aerospace Sciences Meeting including the New Horizons Forum and Aerospace Exposition., 2011.

[14] K. Kragh, P. Fleming, Rotor speed dependent yaw control of wind turbines based on empirical data., in: Proceedings of 50th AIAA Aerospace Sciences Meetings and Exhibit, American Institute of Aeronautics and Astronautics, 2012.

[15] K. Kragh, M. Hansen, T. Mikkelsen, Improving yaw alignment using spinner based lidar., in: Proceedings of 49th AIAA, Aerospace Sciences Meeting Including the New Horizons Forum and Aerospace Exposition., American Institute of Aeronautics and Astronautics, AIAA, 2011.

[16] D. Schlipf, J. Anger, S. Kapp, O. Bischoff, M. Hofsäß, A. Rettenmeier, M. Kühn, Prospects of optimization of energy production by lidar assisted control of wind turbines., in: Proceedings of European Wind Energy Conference and Exhibition, EWEA., 2011.

[17] P. a. Fleming, P. M. O. Gebraad, S. Lee, J. W. van Wingerden, K. Johnson, M. Churchfield, J. Michalakes, P. Spalart, P. Moriarty, Evaluating techniques for redirecting turbine wakes using SOWFA, Renewable Energy 70 (2014) 211-218.

[18] P. M. O. Gebraad, J. W. van Wingerden, A Control-Oriented Dynamic Model for Wakes in Wind Plants, Journal of Physics: Conference Series 524 (2014) 012186.

[19] M. J. Churchfield, P. Fleming, B. Bulder, S. M. White, Wind Turbine Wake-Redirection Control at the Fishermen's Atlantic City Windfarm, in: Offshore Technology Conference, no. May, Offshore Technology Conference, 2015.

[20] K. a. Kragh, M. H. Hansen, T. Mikkelsen, Improving Yaw Alignment Using Spinner Based LIDAR, AIAA Aerospace Sciences Meeting including the New Horizons Forum and Aerospace Exposition (January) (2011) 1-13.

[21] P. a. Fleming, a.K. Scholbrock, a. Jehu, S. Davoust, E. Osler, a.D. Wright, a. Clifton, Field-test results using a nacelle-mounted lidar for improving wind turbine power capture by reducing yaw misalignment, Journal of Physics: Conference Series 524 (1) (2014) 012002.

[22] A. Scholbrock, P. Fleming, A. Wright, C. Slinger, J. Medley, M. Harris, Field Test Results from Lidar Measured Yaw Control for Improved Yaw Alignment with the NREL Controls Advanced Research Turbine: Preprint. (2014).

[23] G. Svensson, A. A. M. Holtslag, Single column modeling of the diurnal cycle based on CASES99 data GABLS second intercomparison project, 17th Symposium on Boundary Layers and Turbulence (2006) $22-25$.

[24] V. Kumar, J. Kleissl, C. Meneveau, M. B. Parlange, Large-eddy simulation of a diurnal cycle of the atmospheric boundary layer: Atmospheric stability and scaling issues, Water Resources Research 42 (2006) $1-18$.

[25] V. Kumar, G. Svensson, a. a. M. Holtslag, C. Meneveau, M. B. Parlange, Impact of surface flux formulations and geostrophic forcing on large-eddy simulations of diurnal atmospheric boundary layer flow, Journal of Applied Meteorology and Climatology 49 (2010) 1496-1516.

[26] V. Sharma, M. Calaf, M. Lehning, M. B. Parlange, Time-adaptive wind turbine model for an les framework, Wind Energy 19 (5) (2016) 939-952.

[27] P. Y. Orzag SA, Numerical computation of turbulent shear flows, Advances in Geophysics 1(224) (1974) $225-236$.

[28] E. Bou-Zeid, C. Meneveau, M. Parlange, A scale-dependent Lagrangian dynamic model for large eddy simulation of complex turbulent flows, Physics of Fluids 17 (2) (2005) 1-18.

[29] M. Calaf, M. B. Parlange, C. Meneveau, Large eddy simulation study of scalar transport in fully developed wind-turbine array boundary layers, Physics of Fluids (2011) 23 (12). 
[30] Y.-T. Wu, F. Porté-Agel, Large-eddy simulation of wind-turbine wakes: Evaluation of turbine parametrisations, Boundary-Layer Meteorology 138 (3) (2010) 345-366.

[31] C.-h. Moeng, A Large-Eddy-Simulation Model for the Study of Planetary Boundary-Layer Turbulence, Journal of the Atmospheric Sciences 41(13) (1984) 2052-2062.

[32] J. D. Albertson, M. B. Parlange, Natural integration of scalar fluxes from complex terrain, Advances in Water Resources 23 (3) (1999) 239-252.

[33] M. Frigo, S. G. Johnson, The design and implementation of FFTW3, Proceedings of the IEEE 93 (2) (2005) 216-231.

[34] C. Canuto, M. Y. Hussaini, A. Quarteroni, T. A. Zang, Spectral Methods in Fluid Dynamics, Springer Berlin Heidelberg, Berlin, Heidelberg, 1988.

[35] A Higher-Order Compact Method in Space and Time Based on Parallel Implementation of the Thomas Algorithm, Journal of Computational Physics 161 (1) (2000) 182-203.

[36] M. B. Parlange, W. Brutsaert, Regional shear stress of broken forest from radiosonde wind profiles in the unstable surface layer, Boundary-Layer Meteorology 64 (4) (1993) 355-368.

[37] M. Hultmark, M. Calaf, M. B. Parlange, A New Wall Shear Stress Model for Atmospheric Boundary Layer Simulations, Journal of the Atmospheric Sciences 70 (11) (2013) 3460-3470.

[38] W. Brutsaert, M. B. Parlange, The unstable surface layer above forest: Regional evaporation and heat flux, Water resources research 28 (12) (1992) 3129-3134.

[39] W. Brutsaert, M. B. Parlange, J. H. C. Gash, Neutral humidity profiles in the boundary layer and regional evaporation from sparse pine forest, Annales Geophysicae 7 (1989) 623-630, 7: 623-630.

[40] W. Brutsaert, Hydrology: An Introduction, Cambridge University Press, 2005.

[41] A. C. Fitch, J. K. Lundquist, J. B. Olson, Mesoscale Influences of Wind Farms throughout a Diurnal Cycle, Monthly Weather Review 141 (2013) 2173-2198.

[42] V. Sharma, M. B. Parlange, M. Calaf, Perturbations to the spatio-temporal characteristics of a diurnal abl due to an idealised wind-farm, Boundary Layer Meteorology (Submitted).

[43] M. Calaf, C. Meneveau, J. Meyers, Large eddy simulation study of fully developed wind-turbine array boundary layers, Physics of Fluids 22 (1) (2010) 015110.

[44] [online] (2015). [link].

[45] [online] (2015). [link].

[46] [online] (2015). [link].

[47] E. Simley, L. Y. Pao, P. Gebraad, M. Churchfield, Investigation of the impact of the upstream induction zone on lidar measurement accuracy for wind turbine control applications using large-eddy simulation, Journal of Physics: Conference Series 524 (1) (2014) 012003.

[48] F. Porté-Agel, Y.-T. Wu, C.-H. Chen, A Numerical Study of the Effects of Wind Direction on Turbine Wakes and Power Losses in a Large Wind Farm, Energies 6 (10) (2013) 5297-5313.

[49] H. Lu, F. Porté-Agel, On the Impact of Wind Farms on a Convective Atmospheric Boundary Layer, Boundary-Layer Meteorology 157 (1) (2015) 81-96.

[50] B. Witha, G. Steinfeld, M. Dörenkämper, D. Heinemann, Large-eddy simulation of multiple wakes in offshore wind farms, Journal of Physics: Conference Series 555 (1) (2014) 012108.

[51] W. Gutierrez, G. Araya, S. Basu, a. Ruiz-Columbie, L. Castillo, Toward Understanding Low Level Jet Climatology over West Texas and its Impact on Wind Energy, Journal of Physics: Conference Series 524 (2014) 012008.

[52] N. G. Nygaard, Wakes in very large wind farms and the effect of neighbouring wind farms, Journal of Physics: Conference Series 524 (1) (2014) 012162.

[53] R. J. Barthelmie, S. T. Frandsen, O. Rathmann, K. Hansen, E. S. Politis, J. Prospathopoulos, J. G. Schepers, K. Rados, Flow and wakes in large wind farms : Final report for UpWind WP8 Ris $\varnothing-\mathrm{R}-$ Report, Vol. Risø-R-Rep, (2011).

[54] H. Lu, F. Porté-agel, Large-eddy simulation of a very large wind farm in a stable atmospheric boundary layer, Physics of Fluids 23 (6) (2011) 065101.

[55] F. Porté-Agel, H. Lu, Y. Wu, Interaction between Large Wind Farms and the Atmospheric Boundary Layer, Procedia IUTAM 10 (0) (2014) 307-318. 